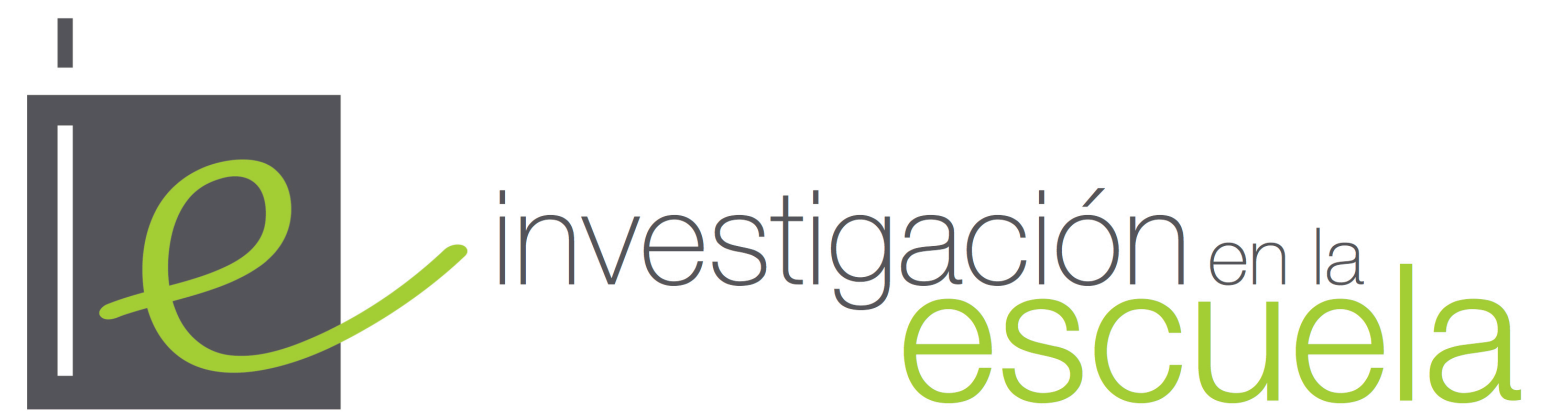

Revista internacional de investigación e innovación educativa

\title{
Valoración por los estudiantes del Grado de Educación Infantil del trabajo por proyectos desde una propuesta de innovación docente interdisciplinar
}

Esther Garcia-González, José M Mardeñoso, Rafael Crismán-Pérez, Lourdes Aragón, Carmen González, Natalia Jiménez, Francisco Moreno, Pilar Azcárate, Susana Sánchez.

Universidad de Cádiz

España

Citación: García-González, E., Cardeñoso, J. M., Crismán-Pérez, R., Aragón, L., González, C., Jiménez, N., Moreno, F., Azcárate, P., Sánchez, S. (2017). Valoración por los estudiantes del Grado de Educación Infantil del trabajo por proyectos desde una propuesta de innovación docente interdisciplinar. Investigación en la Escuela, 91, 19-35. Recuperado de http://www.investigacionenlaescuela.es/articulos/R91/R91-2

Resumen: Presentamos los resultados obtenidos sobre la valoración de los estudiantes, tras el desarrollo de un proyecto de innovación docente interdisciplinar en la Universidad de Cádiz. Este se centraba en la enseñanza-aprendizaje del Trabajo por Proyectos como contenido y como estrategia. En el estudio participaron 123 alumnos. A través de un cuestionario Likert, se caracterizaron dos factores de valoración de la experiencia: el aprendizaje de los aspectos claves curriculares en Educación Infantil a partir del trabajo con proyectos y la incidencia de la innovación docente desarrollada por el profesorado como elemento de aprendizaje. Los resultados obtenidos mostraron una valoración positiva en ambos factores. Asimismo, los alumnos destacaron las estrategias de 
aprendizaje cooperativo, la relevancia de la retroalimentación y la autonomía para la autogestión de las tareas.

Palabras clave: "Trabajo por Proyectos"; "Innovación Educativa"; "Interdisciplinar"; "Formación de Docentes"; "Educación Infantil".

Works with project assessment conducted by students of early childhood education from an interdisciplinary teaching innovation proposal

Abstract: We present the results obtained in the evaluation of the students after the development of an interdisciplinary teaching innovation proposal at the University of Cádiz. This focused on the teaching-learning process of working with projects applied as content and strategy. The present study has involved 123 students. Two factors of the innovation proposal were evaluated through a Likert scale questionnaire: the learning of the key curricular aspects of Child Education working with projects, and the incidence of teacher innovation as a key to the learning process. The results showed a positive valuation in the above mentioned factors. The students emphasized the strategies of cooperative learning, the relevance of feedback, and the autonomy for self-management of tasks. Key words: "Working Through Projects"; "Educational Innovation"; "Interdisciplinary"; "Teacher Education"; "Early Childhood Education".

Évaluation faite par les élèves de grade d'Éducation Maternelle du travail par projet à partir d'une proposée d'innovation d'enseignement interdisciplinaire

Resumè: On présente les résultats de l'évaluation faite par des élèves à propos d'un projet d'innovation d'enseignement à l'Université de Cádiz. Ce projet est concentré sur le procès d'enseignement-apprentissage du travail par projets, comme un contenu et comme une stratégie. L'étude a porté sur 123 étudiants, et il est centré sur deux facteurs d'évaluation de l'expérience grâce à un questionnaire d'échelle Likert : l'apprentissage des aspects clés du curriculum de l'Éducation Maternelle à travers de projets et l'influence de l'innovation éducative développée par le enseignants comme clé dans l'apprentissage. Les résultats obtenus ont montré une évaluation positive, les étudiants ont mis en évidence les stratégies de l'apprentissage coopératif, l’importance de la rétroaction et l'autonomie pour l'autogestion des tâches.

Mostsclé: "Projets de travail"; "Innovation éducative"; "Interdisciplinaire"; "Formation des enseignants"; "Éducation Maternelle".

\section{Introducción}

Presentamos la valoración de los estudiantes que han participado en un proyecto de innovación y mejora docente, desarrollado por un equipo multidisciplinar de profesores de la Facultad de Ciencias de la Educación de la Universidad de Cádiz ${ }^{1}$. La finalidad de dicho proyecto es el tratamiento de la enseñanza-aprendizaje del Trabajo por Proyectos, tanto como estrategia metodológica como también como contenido de enseñanza, manteniendo como estrategia metodológica el Aprendizaje Cooperativo (Aragón et al., 2014).

Durante el desarrollo del mismo se llevaron a cabo diferentes actividades, tanto individuales como grupales, de forma coordinada por todo el equipo de docentes. Una parte fundamental contemplada en el proceso fue la destinada a la evaluación/regulación del mismo. Se trataba de obtener información para valorar el proceso seguido y asimismo poder influir en la toma de decisiones de futuros diseños didácticos (Sanmartí, 2007). Pretendíamos implicar a los estudiantes en

\footnotetext{
${ }^{1}$ Proyecto de Innovación financiado por la Unidad de Innovación Docente de la UCA.
} 
su propio proceso de evaluación, puesto que ello conlleva mejoras tanto en su aprendizaje como en el desarrollo de estrategias profesionales e interpersonales (Vallés, Ureña y Ruiz, 2011).

Durante el proceso se recabó información de las producciones e ideas de todos los participantes en diferentes momentos y, antes de finalizar el proyecto, se invitó a los estudiantes a manifestar su opinión acerca de la experiencia vivida durante el desarrollo del proyecto de innovación. Para ello, empleamos dos instrumentos: un cuestionario individual destinado a la autoevaluación del aprendizaje, previamente validado (Sans, 2008; Muñiz y Fonseca, 2008; Jiménez et al., 2014), y un segundo cuestionario, tipo Likert, que recogía cuestiones relacionadas con la potencialidad de la metodología de trabajo seguida para su futura labor profesional. Sobre los datos obtenidos con este segundo instrumento se centra este artículo.

\title{
Finalidades del proyecto y descripción de los principios metodológicos
}

\author{
Como indican Azcárate, Hamed y Martín del Pozo (2013, p. 49)
}

hay un amplio consenso entre los investigadores sobre la necesidad de diseñar, aplicar y evaluar estrategias formativas con las que los futuros maestros aprendan a cuestionar sus planteamientos en relación con los problemas curriculares fundamentales (qué enseñar y para qué, qué hacer con las ideas que tienen los alumnos, qué tareas poner y cómo hacer el seguimiento, etc.), como paso previo para iniciar cambios sólidos.

El proyecto de innovación y mejora docente universitaria desarrollado intenta dar un paso en esta línea. Presenta una propuesta de trabajo coordinado en el diseño, desarrollo y evaluación desde las cuatro asignaturas de las didácticas específicas impartidas en el $3^{\circ}$ curso del Grado de Maestro en Educación Infantil (GMEI). Las asignaturas implicadas fueron Didáctica para el desarrollo de las habilidades comunicativas escrita, El desarrollo del conocimiento matemático en educación infantil, Didáctica del medio natural y Didáctica de las ciencias sociales.

El diseño se basa en el Trabajo por Proyectos y, en consonancia con la fundamentación de los mismos (Pujol, 1991; Maldonado, 2008), mantiene como estrategia metodológica el Aprendizaje Cooperativo. Se unen así dos elementos complementarios en el aprendizaje de los futuros maestros de Educación Infantil (EI): la comprensión del desarrollo y puesta en práctica del aprendizaje por proyectos, y los procesos de negociación y construcción que supone el trabajo cooperativo. La propuesta tiene como objetivo el trabajo por proyectos, como contenido formativo y como estrategia metodológica del propio proceso formativo.

Abordar el conocimiento profesional implicado es condición sine qua non de toda práctica cuya finalidad es la formación de futuros maestros. El dominio del conocimiento es el que permite y facilita la intervención crítica, autónoma y la toma de decisiones en su contexto profesional, pero su tratamiento no es un fin en sí mismo, sino un medio para promover el desarrollo profesional. Necesitamos profesionales competentes en el diseño, desarrollo y regulación de los procesos educativos en el ámbito de la EI.

En este sentido, en la formación de docentes no solo son relevantes los conocimientos profesionales que intentamos poner en juego, sino que es especialmente importante la manera en que dichos conocimientos son mostrados y tratados en el aula (Azcárate y Cardeñoso, 2011). Desde nuestra perspectiva, ser competente profesionalmente implica ser capaz de organizar y usar los conocimientos disponibles con flexibilidad y aplicarlos en la resolución de los problemas y situaciones que surgen en los diferentes contextos donde cada uno desarrolla su actividad profesional como educador. En esta línea, la metodología constituye un factor vital para el desarrollo de las competencias profesionales. 
El aprendizaje universitario basado en las competencias supone un intento de aunar la formación académica (adquisición y construcción de saberes o conocimientos) y el desarrollo personal y profesional (competencias generales y profesionales). La integración sinérgica de ambos aspectos conlleva una potenciación mutua y permite la puesta en juego de todos los recursos del individuo para dar respuestas a las diferentes situaciones con que se encontrarán en su vida universitaria y profesional. Para el logro de estas competencias se plantearon una serie de actividades formativas entre las que destacamos, por su carácter transversal, el trabajo por proyectos cooperativos. Consideramos, como indican Ruiz y Mérida (2015), que los objetivos y contenidos propios de la EI se integran bien en el desarrollo de proyectos de trabajos realizados desde temáticas vinculadas al interés de los niños (Oliveras et al., 2008); es decir, a aquello que han de aprender y conectadas con su entorno.

Desde estas dos perspectivas optamos, como ya hemos indicado, por el diseño de una estrategia metodológica basada en proyectos. Algunas de las razones en la que se apoya esta decisión son:

- Los proyectos son propuestas pedagógicas que buscan superar la mera transmisión de información del formador/docente al grupo.

- Se acerca al modelo de constructivismo social a partir de una gestión del aula en la que se da cabida a la libre iniciativa de los estudiantes/alumnos y sus interacciones, con el fin de lograr la construcción conjunta del conocimiento.

- Se otorga una gran atención a la comunicación, entendida como acción conjunta aplicada a la resolución de problemas, retos, dudas y deseos de cambio desde diferentes perspectivas y puntos de vista, ajustando la respuesta de una manera más equitativa.

- Se ajustan plenamente a la metodología recomendada para la etapa de EI, donde los egresados desarrollarán su vida profesional (Hernández, 2001).

En la misma línea, la evaluación del proceso se desarrolló mediante un diseño que integraba todos los procesos conjuntos de las actividades desarrolladas, complementado con la evaluación de cada asignatura y la información procedente de los instrumentos de evaluación, coevaluación y autoevaluación de la actividad conjunta.

La clave de la coordinación desarrollada por el equipo de los docentes implicados gira en torno a la propuesta de elaboración de un proyecto para EI, común para las cuatro asignaturas, y tener el aprendizaje cooperativo como estrategia para promover el aprendizaje de los estudiantes y su propio desarrollo profesional. En este sentido, las mejoras que se pretenden con esta experiencia innovadora se enmarcan, en la coordinación de la actividad académica desarrollada en las cuatro asignaturas que configuran la formación didáctica del $3^{\circ}$ curso del GMEI, en relación a:

- Diseño de propuestas metodológicas coherentes con el aprendizaje por proyectos cooperativos para optimizar el aprendizaje.

- Diseño de un sistema de evaluación coherente con el diseño metodológico.

\section{Descripción de la secuencia didáctica}

Una aportación especialmente relevante de esta iniciativa docente es promover que los futuros maestros de EI comprendan, en la medida de lo posible, la naturaleza de los procesos de enseñanza-aprendizaje y el funcionamiento del aula de EI. La importancia que adquiere el diseño de una propuesta de intervención basada en el desarrollo de un proyecto de trabajo para el aula de EI, comunes para las cuatro asignaturas, exige varias cuestiones a los estudiantes. En primer lugar, tienen que vivenciar las actividades derivadas del diseño y desarrollo de dichos proyectos y su posterior análisis didáctico, reflexionando sobre los conocimientos puestos en juego y aprendidos. 
En segundo lugar, se les solicita que se sitúen en la perspectiva del niño de infantil, que aprende. Desde esa perspectiva deben adaptar su proyecto y tomar decisiones sobre cómo presentar y tratar los contenidos de los distintos ámbitos del currículo de EI, de acuerdo a las orientaciones metodológicas apropiadas para promover el aprendizaje. De esta manera, se intenta que el estudiante universitario sea capaz de extrapolar los aprendizajes a contextos de su futura actuación docente de forma significativa y funcional. Se pretende que aprendan haciendo y viviendo.

Como ya hemos indicado, la propuesta se ha centrado en una estructura de diseño común para las cuatro asignaturas desarrolladas en los 3 grupos. Los estudiantes se organizaron en grupos de 4-6, de manera que cada grupo gozó de autonomía para autogestionar las respectivas tareas que se les encomendaron. A lo largo del proceso formativo, se combinaron distintas estrategias y actividades que promueven un aprendizaje cooperativo. Tanto a través de la reflexión individual sobre las diferentes informaciones puestas en juego como por el continuo contraste entre iguales y con el profesorado. En la tabla 1 presentamos un esquema de la secuencia metodológica llevada a cabo.

Tabla 1

Organización común del proceso de enseñanza aprendizaje

Primer momento. Se organizó una actividad conjunta para introducir a los estudiantes en el trabajo por proyectos. Concretada a través de la lectura, análisis y debate sobre el sentido y organización del trabajo por proyectos. Se desarrolló a lo largo de todas las sesiones prácticas y teóricas de las cuatro asignaturas de forma coordinada durante las primeras semanas del curso.

Segundo Momento. Corresponde a gran parte del desarrollo del curso. Se centraron en el desarrollo en paralelo de las sesiones teóricas y prácticas de las cuatro asignaturas. Las teóricas se dedicaron al trabajo propio sobre los contenidos en cada una de ellas y las sesiones prácticas, al diseño y desarrollo de un proyecto a nivel de adultos y su posterior análisis didáctico.

Sesiones teóricas (actividad específica de cada una de las cuatro asignaturas)

a) Estudio y análisis del conocimiento. Se trabajó en cada asignatura el conocimiento específico de la materia, analizando la pro organización y características del currículo de E.I.

b) Estudio sobre las características propias del aprendizaje infantil. En cada asignatura se trataron las características propias del aprendizaje de cada ámbito del conocimiento implicado, sus condiciones de desarrollo y sus peculiaridades en E.I.

c) Estudio de las situaciones de aprendizaje y estrategias de enseñanza. En cada una de las
Sesiones prácticas (actividad común a las cuatro asignaturas)

a) Elección del proyecto. Se procedió a la elección de uno de los siguientes proyectos:

- Basuras en mi entorno

- Mercadillo solidario

- Museo del juguete

- Turista en mi tierra

- Nuestro parque ideal

- Laboratorio de aromas

- Comienza la emisión

b) Diseño y desarrollo y puesta en práctica del proyecto desde el punto de vista del adulto implicado: Esta actividad 


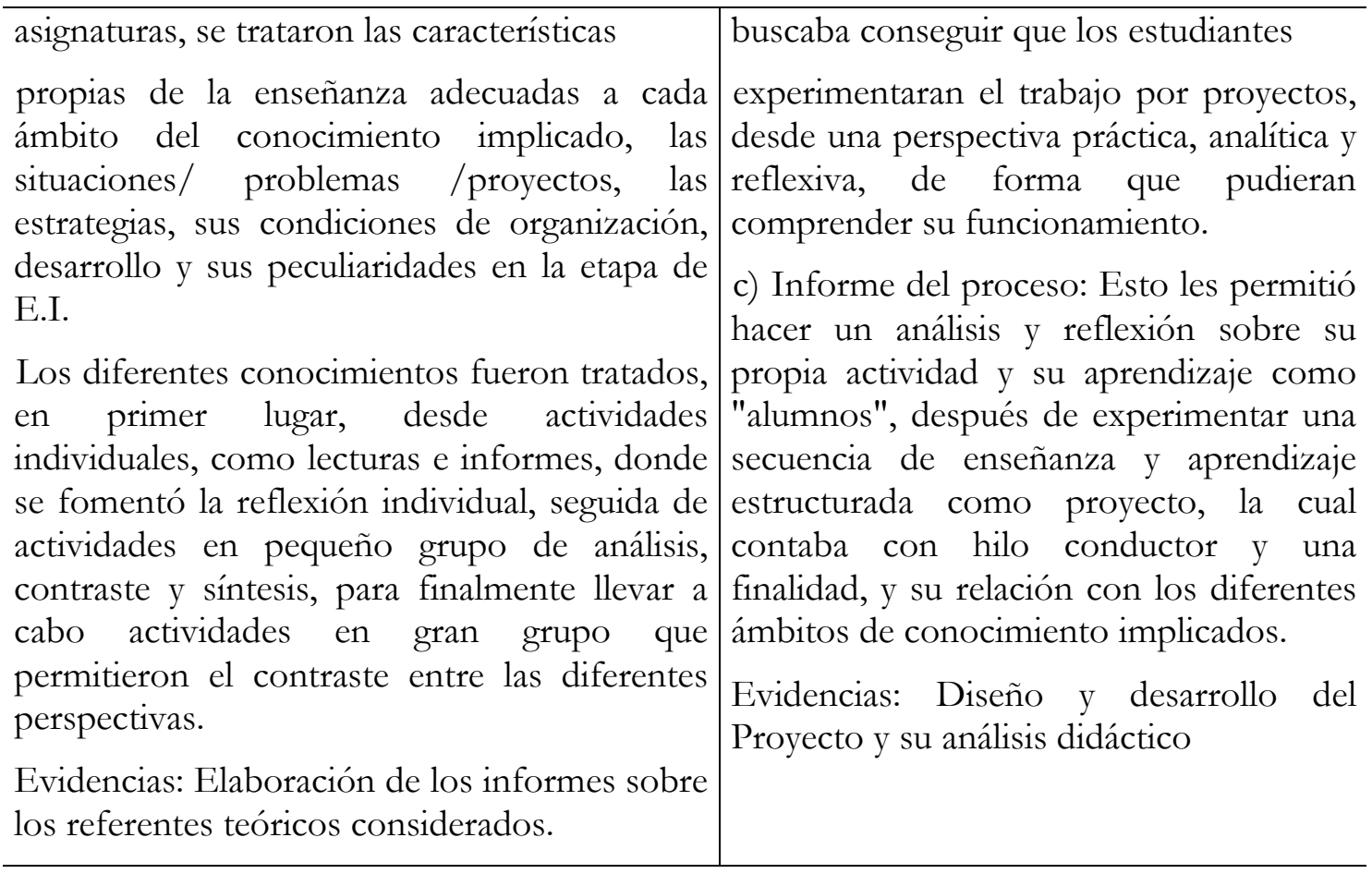

Tercer momento. Adaptación del proyecto elaborado a un aula de Educación Infantil. Esta propuesta final consistió en trasladar el proyecto desarrollado previamente a nivel adulto a un aula de infantil concreta, de forma argumentada y apoyada en los referentes teóricos elaborados. Constó de dos apartados, uno específico para cada asignatura, y uno común a las cuatro:

\section{Evidencias:}

- Planteamiento y descripción del proyecto y la secuencia de actividad diseñada adaptada para un aula de $2^{\circ}$ ciclo de E.I. Este apartado es común a las cuatro asignaturas

Análisis didáctico de la propuesta de intervención en función de los conocimientos implicados en el desarrollo del proyecto. Específico para cada asignatura.

\section{Método}

\section{Participantes}

Para la realización de esta investigación se ha contado con un total de 123 estudiantes matriculados en el curso de $3^{\circ}$ del GMEI, correspondientes a las tres líneas del citado curso. De los cuales solo el 10\% eran varones y la edad media estaba en torno a los 21.3 años.

\section{Variables e instrumentos}

Las variables de estudio fueron seleccionadas de acuerdo a una serie de investigaciones especializadas en esta cuestión (León y López, 2014; López, 2005; Mauri, Coll y Onrubia, 2007; Ortega et al., 2007) y se concretaron en dos factores: 
- Factor 1. La eficacia de la metodología por proyectos como factor de organización escolar en el proceso de enseñanzaaprendizaje. Para la valoración de esta variable tuvimos en cuenta las propuestas acerca de la metodología de aprendizaje desde una perspectiva corporativa y colaborativa (Pujolás y Lago, 2007; Riera, 2011) en un contexto de trabajo en equipo.

Diseñamos un conjunto de ítems, con respuesta tipo escalas Likert de 5 valores, donde los estudiantes debían hacer una evaluación a partir de diferentes ítems de la metodología de trabajo llevada a cabo. Recurrimos a literatura científica especializada para la validación de la escala (Ruys, Van Keer y Aelterman, 2014; Taylor y Burke, 2014). A partir de ahí, elaboramos un instrumento adaptado al contexto concreto en el que habíamos desarrollado nuestra innovación. La escala inicial fue de una batería de 55 ítems.

- Factor 2. La innovación docente como factor de mejora del aprendiaje del alumnado. El segundo factor que tuvimos en cuenta fue la valoración por parte del alumnado de la innovación docente desarrollada y sus repercusiones en el aprendizaje. Para ello elaboramos igualmente, desde la literatura, un conjunto de ítems presentados mediante una escala Likert que, en un principio, contempló una batería también de 55 ítems. Todos los ítems de los instrumentos iniciales fueron sometidos a un criterio de supervisión por jueces que nos permitió establecer una gradación de cinco elementos para cada uno de los factores que pretendimos medir.

Para la validación de los instrumentos, recurrimos a un proceso de análisis estadístico de las escalas a partir de un muestreo que consistió en administrar el instrumento a cincuenta sujetos de la muestra global y, consecuentemente, llevar a cabo un análisis factorial exploratorio. Una vez hecho esto, recurrimos a un proceso de selección de los ítems con mayor poder discriminante basándonos en la carga factorial menor de 0.4 y se descartó aquellos ítems ambiguos desde el punto de vista factorial. Seguidamente, realizamos un análisis factorial confirmatorio a partir del descarte de ítems antes dicho. De esta manera, la varianza total explicada mediante el método Varimax fue del $40.576 \%$.

Finalmente, obtuvimos un instrumento de 25 ítems con sus valores entre 1-5, correspondiente a los dos factores confirmados: la metodología por proyectos como factor de organización escolar en el proceso de enseñanza-aprendizaje (15 ítems) y la innovación docente como factor de mejora del aprendizaje del alumnado (10 ítems), las cuales constituyeron los aspectos a evaluar.

\section{Resultados}

Una vez cumplimentado el cuestionario se procedió a su análisis, considerando los dos factores seleccionados para el estudio.

\section{Factor 1. La eficacia de la metodología por proyectos}

Tras el proceso de análisis estadístico, el primer factor se reflejó en el instrumento a través de un total de 15 ítems, con un alpha de Cronbach de 0.89 .

Tabla 2

Estadísticos descriptivos del factor 1

\begin{tabular}{llllll}
\hline $\mathrm{N}$ & Mínimo & Máximo & Media & Desviación típica & Varianza \\
123 & 1.80 & 4.80 & 3.43 & .7320 & .536 \\
\hline
\end{tabular}


Tabla 3

Estadísticos del primer factor por items

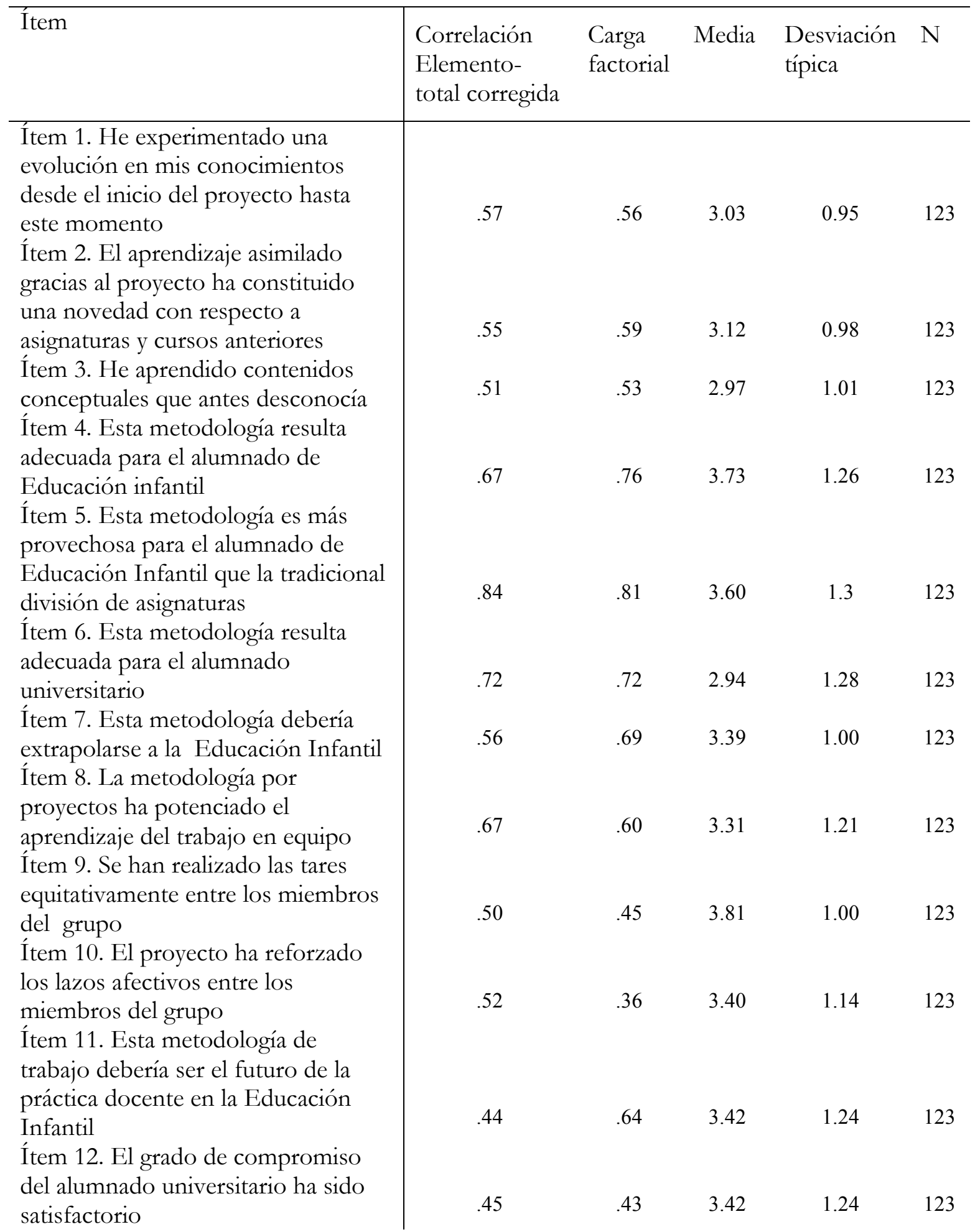


Tabla 3 (Cont.)

Estadísticos del primer factor por items

\begin{tabular}{l|lcccc}
\hline Ítem & $\begin{array}{l}\text { Correlación } \\
\text { Elemento-total } \\
\text { corregida }\end{array}$ & $\begin{array}{l}\text { Carga } \\
\text { factorial }\end{array}$ & Media & $\begin{array}{l}\text { Desviación } \\
\text { típica }\end{array}$ & $\mathrm{N}$ \\
\hline $\begin{array}{l}\text { Ítem 13. Esta metodología docente } \\
\text { por proyectos debería estudiarse más } \\
\text { para su adecuada aplicación al aula } \\
\text { de EI }\end{array}$ & .67 & .42 & 3.80 & 1.05 & 123 \\
$\begin{array}{l}\text { Ítem 14. El profesorado debería } \\
\text { poner más a menudo este tipo de } \\
\text { iniciativas de innovación docente } \\
\begin{array}{l}\text { Ítem 15. La respuesta del alumnado } \\
\text { hacia el proyecto ha sido adecuada }\end{array}\end{array}$ & .57 & .57 & 4.08 & 0.99 & 123 \\
\hline
\end{tabular}

En cuanto al agrupamiento de los ítems, debemos destacar el ámbito de satisfacción del alumnado con esta metodología de trabajo. Se reflejó en la alta valoración de los estudiantes en los ítems que la intentaban recoger (ítems $6,8,9,10,12,14$ y 15), seguido por al ámbito correspondiente al nivel de aprendizaje experimentado (ítems 1, 2 y 3). Es también significativo el reconocimiento de la adecuación del trabajo por proyectos a los procesos educativos en EI (ítems 4, $5,7,11,13)$, con valores todos por encima de la media.

Es significativo que el ítem 14 sea el de mayor valoración (4.08), donde el estudiante refleja su percepción positiva de la experiencia al reclamarla para otras asignaturas. Por otro lado, uno de los aspectos más relevantes lo constituye el ítem 9 con (3.81), referido al trabajo equitativo. Los participantes resaltaron el aprendizaje de estrategias para desarrollar el trabajo en equipo como una de las principales habilidades adquiridas mediante el trabajo por proyectos. Esto corrobora lo expuesto en otras investigaciones (Casasola, Pérez y García, 2012), ya que los participantes manifestaron la potenciación y el aprendizaje de estrategias interpersonales como parte de las competencias activadas durante la realización de las diferentes tareas del proyecto. En este punto, la metodología docente del trabajo por proyectos supuso un estímulo para el aprendizaje de los contenidos tanto procedimentales como actitudinales, los cuales se entrelazaron a los conceptuales de cada didáctica específica implicada.

\section{Factor 2. La innovación docente como factor de mejora del aprendizaje}

Finalmente, tras el proceso de selección de ítems, este factor contó con un total de 10 ítems con un alpha de Cronbach de 0.82 .

Tabla 4

Estadísticos descriptivos del factor 2

\begin{tabular}{llllll}
\hline $\mathrm{N}$ & Mínimo & Máximo & Media & Desviación típica & Varianza \\
123 & 1.50 & 4.70 & 2.97 & .7392 & .546 \\
\hline
\end{tabular}


Tabla 5

Estadísticos del segundo factor por items

\begin{tabular}{|c|c|c|c|c|c|}
\hline Ítem & $\begin{array}{l}\text { Correlación } \\
\text { Elemento- } \\
\text { total } \\
\text { corregida }\end{array}$ & $\begin{array}{l}\text { Carga } \\
\text { factorial }\end{array}$ & Media & $\begin{array}{l}\text { Desviación } \\
\text { típica }\end{array}$ & $\mathrm{N}$ \\
\hline $\begin{array}{l}\text { Ítem 16. La formación recibida en las } \\
\text { asignaturas ha sido fundamental para } \\
\text { entender el Trabajo por proyectos en } \\
\text { E.I. }\end{array}$ & .52 & .47 & 2.70 & 1.03 & 123 \\
\hline $\begin{array}{l}\text { Ítem } 17 \text {. La coordinación del } \\
\text { profesorado entre sí ha sido adecuada }\end{array}$ & .70 & .55 & 2.21 & 1.12 & 123 \\
\hline $\begin{array}{l}\text { Ítem 18. El proyecto debería haber } \\
\text { sido revisado más exhaustivamente } \\
\text { por el profesorado antes de su puesta } \\
\text { en marcha }\end{array}$ & .41 & .32 & 3.99 & 1.05 & 123 \\
\hline $\begin{array}{l}\text { Ítem } 19 . \text { Los criterios e instrumentos } \\
\text { de evaluación del proyecto han sido } \\
\text { adecuados }\end{array}$ & .73 & .64 & 2.82 & 1.04 & 123 \\
\hline $\begin{array}{l}\text { Ítem 20. El tiempo general empleado } \\
\text { durante las sesiones de clase ha sido } \\
\text { adecuado }\end{array}$ & .72 & .64 & 3.30 & 1.21 & 123 \\
\hline $\begin{array}{l}\text { Ítem } 21 \text {. El alumnado ha poseído la } \\
\text { suficiente autonomía y libertad por } \\
\text { parte del profesorado para desarrollar } \\
\text { adecuadamente el proyecto. }\end{array}$ & .42 & .41 & 3.47 & 1.12 & 123 \\
\hline $\begin{array}{l}\text { Ítem 22. El proyecto debería ofrecer } \\
\text { la posibilidad de llevarse a cabo de } \\
\text { manera individual y no únicamente } \\
\text { en grupo }\end{array}$ & .69 & .55 & 2.52 & 1.53 & 123 \\
\hline $\begin{array}{l}\text { Ítem23. Los temas presentados por } \\
\text { parte del profesorado para desarrollar } \\
\text { la metodología proyectos ha sido } \\
\text { adecuado }\end{array}$ & .54 & .45 & 3.10 & 1.10 & 123 \\
\hline $\begin{array}{l}\text { Ítem 24. La estructuración de la } \\
\text { asignatura en dos proyectos ha sido } \\
\text { adecuada }\end{array}$ & .58 & .61 & 2.48 & 1.19 & 123 \\
\hline $\begin{array}{l}\text { Ítem } 25 \text {. Esta metodología de trabajo } \\
\text { debería aplicarse durante los } \\
\text { próximos cursos a otras } \\
\text { especialidades del Grado de Maestro }\end{array}$ & .74 & .66 & 3.07 & 1.40 & 123 \\
\hline
\end{tabular}


Con respecto al factor 2 , el ámbito retroalimentación del proceso de enseñanza-aprendizaje entre alumnado y profesorado es el que acumuló un mayor número de ítems $(17,18,19,20,21,23$ y 24), cuatro de ellos obtuvieron una valoración media positiva. Los estudiantes valoran el trabajo desarrollado por el equipo de formadores y, en todo caso, demandan una mayor cercanía en el seguimiento del proceso realizado, como nos muestra el valor medio de (3.99) en el ítem 18.

Uno de los aspectos más llamativos es el de la retroalimentación en el proceso de enseñanza y aprendizaje entre alumnado y profesorado, a raíz de la autonomía de organización de la que ha gozado cada conjunto de alumnos asignado a una temática de proyecto determinada. Si observamos el ítem 21 del segundo factor, este ha presentado una de las medias más altas (3.47) de todos los ítems de ambos factores, lo que nos informa de la relevancia de la autonomía y libertad concedidas por los docentes a los participantes. Este punto es de especial importancia, ya que en otras investigaciones (Ávila, 2009; Román, 2009; Ruys, Van Keer y Aelterman, 2014) se ha comprobado la decisiva contribución de la retroalimentación como parte del proceso de enseñanza-aprendizaje.

\section{Discusión de resultados}

Según los estadísticos descriptivos obtenidos, el factor 1, $(\mathrm{X}=3.43)$, presenta una puntuación media superior al factor $2,(\mathrm{X}=2.97)$ que le da una mayor representatividad.

De la revisión de los datos obtenidos globalmente, podemos destacar la dificultad de los participantes para percibir la coordinación del profesorado implicado en el proyecto. Así el ítem 17, del segundo factor, ha obtenido la media más baja con (2.21). No obstante, los estudiantes se muestran a favor de este tipo de iniciativas de innovación docente, por parte del profesorado, como demuestra el ítem 15 del factor 1, con una media de (3.44). Asimismo, han reflejado la carga de trabajo que esto genera como aspecto negativo. Este ha sido el punto peor valorado de la experiencia: la puesta en marcha de nuevas metodologías docentes supone a priori una adaptación a las mismas por parte de docentes y discentes, lo cual conlleva una reorganización de las tareas y técnicas de aprendizaje y, consecuentemente, una dificultad añadida al itinerario habitual de trabajo personal y grupal.

Sin embargo, hemos podido constatar el interés que despierta la puesta en práctica de este tipo de proyectos de innovación docente, debido a la aportación de los mismos a la formación global del alumnado universitario. En nuestro caso, supuso trascender la tradicional enseñanza autónoma, basada en el aislamiento de materias, con producciones parciales desde didácticas tratadas monolíticamente, hacia un aprendizaje basado en la motivación desde una perspectiva profesional y orientadora, más allá del discurso doctrinal (Azcárate et al., 2014) y sectario de carácter academicista de cada didáctica específica.

De este modo, como hemos podido comprobar, la experiencia resultó beneficiosa para el aprendizaje de los estudiantes participantes en el proceso, especialmente las estrategias de trabajo en equipo, así como por la retroalimentación del proceso como evaluación formativa. Hecho que influyó en su comprensión vivenciada de la teoría constructivista para el aula de infantil y en el desarrollo de capacidades y habilidades interpersonales. El alumnado participante manifestó la mejora de los conocimientos globales acerca del carácter formativo de la metodología por proyectos en el aula del GMEI, a partir de la asimilación no solo de contenidos de ámbito didácticoconceptual, sino de estrategias que habilitan al estudiante para la autogestión de su aprendizaje. En este punto, planteamos que la universidad debe proporcionar, como institución básica para los futuros profesionales de la EI, estrategias y destrezas para desarrollar adecuadamente la autogestión del propio aprendizaje. En la misma línea debe promover la interacción del trabajo en equipo a 
partir tanto de las prácticas institucionales del alumnado como de los contenidos impartidos en las diferentes asignaturas que componen su currículo; la sinergia de ideas resulta de enorme relevancia en esta etapa de formación, como futuros profesionales (Martín y Jiménez, 1992; Limón, 1994; Bassedas, Huguet y Solé, 1998).

Los estudiantes manifestaron una valoración positiva hacia esta metodología de trabajo cooperativo tanto en su rol de alumno como en su rol de futuro docente, mediante el cual experimentaron una metodología de trabajo en equipo a la hora de llevar a cabo las diferentes fases en las que se estructuró el proyecto, así como en la toma de decisiones para autogestionarlo. De nuevo, este enfoque confirma lo expuesto en otras investigaciones (Young y Morgan, 2015) acerca del intercambio de experiencias entre estudiantes y profesionales sobre la metodología de trabajos por proyectos como factor clave de la formación del profesorado. En este sentido, este tipo de vivencias constituyen un aprendizaje experimental para el estudiante y un complemento para su proceso de formación como maestro de EI; así como un intercambio de conocimientos a partir de las diferentes temáticas abordadas en el conjunto de proyectos asignados a cada grupo.

\section{Conclusiones}

En primer lugar, podemos concluir que los participantes del proyecto valoraron globalmente de manera positiva la organización metodológica a partir del trabajo por proyectos en un aula de formación, así como la innovación docente para su aprendizaje.

El proceso de coordinación entre el profesorado que compuso el equipo docente y la posterior comunicación que se estableció con los estudiantes participantes del proyecto resultó fundamental para un adecuado proceso de enseñanza-aprendizaje, en el cual las diferentes fases del proyecto (véase tabla 1) estuvieron en continua revisión, tanto por parte del alumnado como del profesorado. Esto propició una retroalimentación constante entre ambos agentes, lo que convergió en un proceso simultáneo de aprendizaje y de evaluación formativa durante todo el proceso de realización del proyecto de innovación. De esta manera, como han resaltado otros trabajos (Boud, Lawson y Thompson, 2014; Castañeda y Adell, 2014), esta continua intercomunicación, a partir de diferentes instrumentos de evaluación a lo largo de un proceso global de aprendizaje entre los estudiantes participantes del proyecto entre sí, así como la comunicación entre estos y los profesores de referencia, potenció la coparticipación del proceso de enseñanza y aprendizaje y, consecuentemente, proyectó el enfoque innovador de la metodología docente, a partir de la simultaneidad de los procesos de formación y evaluación retroalimentados.

Esto confirma los beneficios de los proyectos de innovación docente para el aprendizaje del estudiante universitario y, de nuevo, ofrece evidencias de validez al segundo factor que hemos evidenciado en la presente investigación, ratificando la relevancia de poner en marcha proyectos de innovación docente que ofrezcan nuevas formas de abordar el proceso de enseñanza y aprendizaje en el ámbito universitario adaptados a las nuevas necesidades de formación del alumnado. Al tiempo que estas iniciativas sirvan para retroalimentar el propio proceso de formación del profesorado universitario, el cual, como apuntan otros investigadores (Torelló, 2011), ha transformado su rol tradicional en el proceso de enseñanza. De este modo, los proyectos de innovación docente suponen una herramienta para la mejora de la práctica educativa en la universidad, al constituir ejemplos reales de optimización y mejora de la práctica, así como de iniciativas para la coordinación y el enriquecimiento docente (Castilla, 2011).

Finalmente, nos gustaría destacar la convicción alcanzada por los estudiantes respecto del trabajo por proyectos como ejemplo de aprendizaje integrador de diversos conocimientos a partir de 
una temática determinada, de carácter socio-cultural, lo que también se ve corroborado por otras investigaciones (Machado, 2007). Esto es de vital importancia, ya que constituye un ejemplo real de cómo llevar a cabo la integración de contenidos, tanto para diseñar la intervención en EI como para la coordinación docente, donde se constata que el medio es el mensaje, evidenciando el principio de isomorfía entre la teoría desarrollada y la práctica en el aula (Azcárate y Cardeñoso, 2011).

De este modo, se ha comprobado cómo estos estudiantes universitarios han experimentado mediante el rol de estudiantes esta metodología docente, lo cual les ha permitido construir nuevos referentes prácticos. Y el profesorado universitario ha desarrollado estrategias de coordinación, seguimiento y evaluación. Por otra parte, la participación del estudiantado en la metodología de trabajo por proyectos, adquiriendo el rol del profesorado en el desarrollo de la trasposición didáctica del proyecto para un aula de infantil, ha supuesto un intercambio de los tradicionales roles de estudiante y profesorado (Prieto, Mijares y Llorent, 2014).

En definitiva, consideramos la innovación docente en el ámbito universitario no solo como herramienta de mejora del aprendizaje del estudiante, sino también como recurso de desarrollo del propio docente universitario a partir de las oportunidades que ofrece el Marco del Espacio Europeo de Educación Superior (Margalef y Álvarez, 2005; Monreal, 2009).

\section{Referencias}

Aragón, C., Azcárate, P., Cardeñoso, J.M., Moreno F., Sánchez, S., Jiménez, N. \& Aragón, L. (2014). Una propuesta integradora para la formación inicial de maestros de educación infantil. Comunicación presentada En IV Jornadas de Innovación Docente. Abriendo caminos para la mejora educativa. Sevilla: Facultad de Ciencias de la Educación.

Ávila P. (2009). La importancia de la retroalimentación en los procesos de evaluación. Una revisión del estado del arte. México: Universidad del Valle de México. Disponible en: http://scopeo.usal.es/usodidactico-del-podcast-para-la-retroalimentacion-en-la-docenciauniversitaria/\#sthash.aMSOSwJ0.dpuf.

Azcárate, P. \& Cardeñoso, J.M. (2011). La enseñanza de la estadística a través de escenarios: implicación en el desarrollo profesional. Bolema, 24(40), 789-810.

Azcárate, P., Hamed, S. \& Martín del Pozo, R. (2013). Recurso formativo para aprender a enseñar ciencias por investigación escolar. Investigación en la Escuela, 80, 49-66

Bassedas, E., Huguet, T. \& Solé, I. (1998). Aprender y enseñar en Educación Infantil. Barcelona: Graó.

Boud, D., Lawson, R. \& Thompson, D.G. (2014). The calibration of student judgment through selfassessment: disruptive effects of assessment patterns. Higher Education Research \& Development, 34(1), 45-59.

Casasola, M.A., Pérez, V.A. \& García, J. (2012). Aprendizaje basado en proyectos y trabajo en equipo: innovando en la docencia de la asignatura "sistemas contables informatizados". Revista UPO Innova, 1, 107-122.

Castañeda, L. \& Adell, J. (2014). Beyond the tools: analyzing personal and group learning environments in a university course. Cultura y educación, 26(4), 739-774. doi: 10.1080/11356405.2014.985946.

Castilla, F. (2011). Calidad docente en el ámbito universitario: un estudio comparativo de las universidades andaluzas. Educade, 2, 157-172.

Hernández, F. (2001). Los proyectos de trabajo. Cuadernos de Pedagogía, 310, 79-82.

Jiménez, N., Aragón, L., Sánchez, S., Aragón, C., Azcárate, P., Cardeñoso, J.M. \& Moreno, F. (2014). La coevaluación/autoevaluación como instrumentos para valorar la competencia en el 
trabajo de equipo. Comunicación presentada en IV Jornadas de Innovación Docente. Abriendo caminos para la mejora educativa. Sevilla. Facultad de Ciencias de la Educación Universidad de Sevilla.

León, M.J. \& López, M.C. (2014). Criterios para la evaluación de los proyectos docentes universitarios. Estudios sobre educación, 26, 79-101.

Limón, M.R. (1994). Valores sociales y trabajo en equipo en Educación Infantil. Revista Complutense de Educación, 5(1), 109-120.

López, F. (2005). Metodología participativa en la enseñanza universitaria. Madrid; Narcea.

Machado, A.M. (2007). Un enfoque globalizador en Educación Infantil. Cuadernos de docencia. Revista digital de educación, 1, 1-5.

Maldonado, M. (2008). Aprendizaje Basado en Proyectos Colaborativos Una experiencia en educación superior. Laurus, 14(28), 158-180

Margalef, A. \& Álvarez, J.M. (2005). La formación del profesorado universitario para la innovación en el Marco de la Integración del Espacio Europeo de Educación Superior. Revista de Educación, 337, 51-70.

Martín, M.C. \& Jiménez, J.R. (1992). El trabajo en grupo en Educación Infantil. Aula de Innovación Educativa, 9, 25-28.

Mauri, T., Coll, C. \& Onrubia, J. (2007). La evaluación de la calidad de los procesos de innovación docente universitarios. Una perspectiva constructivista. Revista de docencia universitaria, 1. Disponible en: http://red-u.net/redu/files/journals/1/articles/47/public/47-36-2-PB.pdf.

Monreal, M.C. (2009). La Formación del Profesorado en el marco de la innovación docente universitaria. El caso de la Universidad Pablo de Olavide. Revista de docencia universitaria, 4, 1 12. Disponible en http://red-u.net/redu/files/journals/1/articles/114/public/114-100-2PB.pdf.

Muñiz, J. \& Fonseca, E. (2008). Construcción de instrumentos de medida para la evaluación universitaria. Revista de investigación en educación, 5, 13-25.

Oliveras, M.L., Cardeñoso, J.M., Molina, M. \& Servín, C.Y. (2008). Use of Integrated Projects in a Mathematics Education Course for Prospective Kindergarten Teachers. The International Journal of Interdisciplinary Social Sciences, 2(6), 165-174.

Ortega, P., Ramirez, M.E., Torres, J.L., López, A.E., Sevín, C.Y., Suárez, L. \& Ruiz, B. (2007). Modelo de innovación educativa. Un marco para la formación y el desarrollo de una cultura de la innovación. Revista Iberoamericana de Educación a Distancia, 10(1), 145-173.

Prieto, M.; Mijares, B. \& Llorent, V. (2014). Roles del docente y del alumnado universitario desde las perspectivas de ambos protagonistas del hecho educativo. Revista Electrónica de Humanidades, Educación y Comunicación Social, 18(9), 273-293.

Pujol, M. (1991). Treballar per projectes a parulari. Barcelona: Eumo Editorial.

Pujolás, P. \& Lago, J.R. (2007). La organización cooperativa de la actividad educativa. En Bonals, J. (Ed.). Manual de asesoramiento psicopedagógico (pp. 349-392). Barcelona: Graó.

Riera, G. (2011). El aprendizaje cooperativo como metodología clave para dar respuesta a la diversidad del alumnado desde un enfoque inclusivo. Revista Latinoamericana de Educación Inclusiva, 5(2), 133-149.

Román Maldonado, C.E. (2009). Sobre la retroalimentación o feedback en la educación superior online. Revista Virtual Universidad Católica del Norte, 26, 1-18.

Ruiz, M.C. \& Mérida, R. (2015). Educar para la diversidad a través de los Proyectos de Trabajo en las aulas infantiles. Un estudio de caso. Investigación en la Escuela, 86, 89-102.

Ruys, I., Van Keer, H. \& Aelterman, A. (2014). Student and novice teacher's about collaborative learning implementation. Teachers and Teaching: Theory and Practice, 20(6), 688-703.

Sanmartí, N. (2007). 10 ideas clave. Evaluar para aprender. Barcelona: Graò. 
Sans, A. (2008). La evaluación de los aprendizajes: construcción de instrumentos. Barcelona: Octaedro.

Taylor, C. \& Burke da Silva, K. (2014). An analysis of the effectiveness of feedback to students on assessed work. Higher Education Research \& Development, 33(4), 794-806.

Torelló, O. (2011). El profesor universitario: sus competencias y formación. Profesorado. Revista de curriculum y formación del profesorado, 15(3), 195-211.

Vallés C., Ureña, N. \& Ruiz, E. (2011). La Evaluación Formativa en Docencia Universitaria. Resultados globales de 41 estudios de caso. Revista de Docencia Universitaria, 9(1), 135-158.

Young, T. \& Morgan, A. (2015). Show me what you know creating classroom projects. The Reading Teacher, 68(5), 388-392.

\section{Información sobre los autores}

Autor: Esther García-González

Institución: Universidad de Cádiz

Email: esther.garcia@uca.es

Autor: José $\mathrm{M}^{\mathrm{a}}$ Cardeñoso

Institución: Universidad de Cádiz

Email: josemaria.cardenoso@uca.es

Autor: Rafael Crismán-Pérez

Institución: Universidad de Cádiz

Email: rafael.crisman@uca.es

Autor: Lourdes Aragón

Institución: Universidad de Cádiz

Email: lourdes.aragon@uca.es

Autor: Carmen González

Institución: Universidad de Cádiz

Email: carmen.aragon@uca.es

Autor: Natalia Jiménez

Institución: Universidad de Cádiz

Email: natalia.jimenez@uca.es

Autor: Francisco Moreno

Institución: Universidad de Cádiz

Email: franciscomanuel.moreno@uca.es

Autor: Pilar Azcárate

Institución: Universidad de Cádiz

Email: pilar.azcarate@uca.es

Autor: Susana Sánchez

Institución: Universidad de Cádiz

Email: susana.sanchez@uca.es 


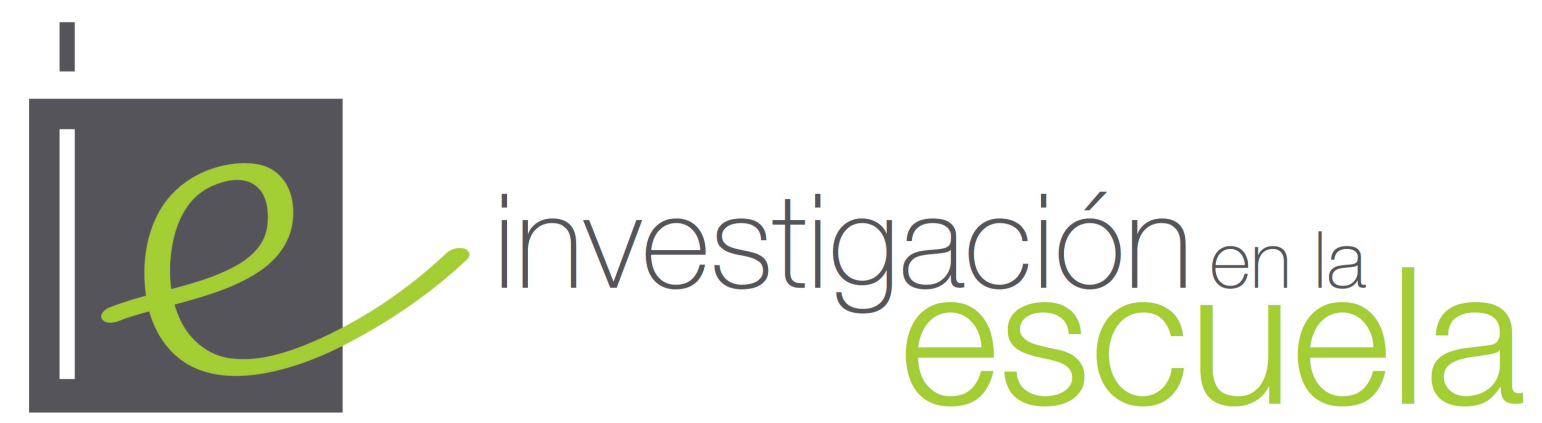

Revista internacional de investigación e innovación educativa

Número 91

06 de julio de 2017

ISSN 2443-9991

(9)

SOMERIIGHISRESEEVEDLOS/as lectores/as pueden copiar, mostrar, y distribuir este artículo, siempre y cuando se de crédito y atribución al autor/es y a Investigación en la Escuela, se distribuya con propósitos no-comerciales, no se altere o transforme el trabajo original. Más detalles de la licencia de CreativeCommons se encuentran en http://creativecommons.org/licenses/by-nc-sa/3.0 Cualquier otro uso debe ser aprobado en conjunto por el autor/es, o Investigación en la Escuela.

ư

Revista Editada por la Universidad de Sevilla. https://editorial.us.es/es/revistainvestigacion-en-la-escuela

Contribuya con comentarios y sugerencias en la web de la revista. Por errores y sugerencias contacte a investigacionescuela@,ddcc.uhu.es 


\section{Investigación en la escuela}

Consejo de dirección: Ana Rivero García (Universidad de Sevilla), Nicolás de Alba Fernández(Universidad de Sevilla), Pedro Cañal de León (Universidad de Sevilla), Francisco F. García Pérez (Universidad de Sevilla), Gabriel Travé González, (Universidad de Huelva), Francisco F. Pozuelos Estrada (Universidad de Huelva)

Dirección: Ana Rivero García y Nicolás de Alba Fernández

Secretaría de edición: Elisa Navarro Medina

\section{Consejo editorial}

José Félix Angulo Rasco. Universidad de Cádiz Rosa Ma Ávila Ruiz. Universidad de Sevilla Pilar AzcárateGoded. Universidad de Cádiz Juan Bautista Martínez Rodríguez. Universidad de Granada

Nieves Blanco García. Universidad de Málaga Fernando Barragán Medero. Universidad de La Laguna José Carrillo Yáñez. Universidad de Huelva José Contreras Domingo. Universidad de Barcelona. Luis C. Contreras González. Universidad de Huelva Ana $\mathbf{M}^{\mathbf{a}}$ Criado García-Legaz. Universidad de Sevilla Rosario Cubero Pérez. Universidad de Sevilla José $\mathbf{M}^{\mathbf{a}}$ Cuenca López. Universidad de Huelva Jesús Estepa Giménez. Universidad de Huelva Rafael Feito Alonso. Universidad Complutense (Madrid)

Francisco José García Gallardo. Universidad de Huelva

Soledad García Gómez. Universidad de Sevilla J. Eduardo García Díaz. Universidad de Sevilla
Fernando Hernández Hernández. Universidad de Barcelona

Salvador Llinares Ciscar. Universidad de Alicante Alfonso Luque Lozano. Universidad de Sevilla Rosa Martín del Pozo. Universidad Complutense (Madrid)

José Martín Toscano. IES Fernando Herrera (Sevilla) Jaume Martínez Bonafé. Universidad de Valencia F. Javier Merchán Iglesias. Universidad de Sevilla Emilia Moreno Sánchez. Universidad de Huelva.Rosario Ortega Ruiz. Universidad de Córdoba

Antonio de Pro Bueno. Universidad de Murcia Fco. de Paula Rodríguez Miranda. Universidad de Huelva

Pedro Sáenz-López Buñuel. Universidad de Huelva Antoni Santisteban Fernández. Universidad Autónoma (Barcelona) Emilio Solís Ramírez. Catedrático de IES. $\mathbf{M}^{\mathbf{a}}$ Victoria Sánchez García. Universidad de Sevilla. Magdalena Suárez Ortega. Universidad de Sevilla

\section{Consejo asesor}

Manuel Área Moreira. Universidad de La Laguna

Jaume Carbonell. Director Cuadernos de Pedagogía. Barcelona

César Coll. Universidad de Barcelona

Christopher Day. Universidad de Nothingham. U.K.

Juan Delval. Universidad Nacional de Educación a Distancia

John Elliott. Universidad de East Anglia. Norwich. U.K.

José Gimeno Sacristán. Universidad de Valencia

André Giordan. Universidad de Paris VII y Ginebra

Francisco Imbernón. Universidad de Barcelona

Ángel Pérez Gómez. Universidad de Málaga

Rafael Porlán Ariza. Universidad de Sevilla

Francesco Tonucci. Instituto de Pedagogía del C.N.R. Roma

Jurjo Torres Santomé. Universidad de A Coruña 
